\title{
Utilizing Game Theory to Optimize In-motion Wireless Charging Service Efficiency for Electric Vehicles
}

\author{
LI YAN, Massachusetts Institute of Technology and University of Virginia \\ HAIYING SHEN, University of Virginia
}

\begin{abstract}
Charger lanes, which are road segments equipped with in-motion wireless chargers, are expected to keep Electric Vehicles (EVs) continuously driving without recharging downtime. To maximize the service efficiency of the in-motion wireless chargers, we must properly coordinate the traffic of the EVs to avoid the generation of congestion at the charger lanes and on the road segments to them. In this article, we propose WPT-Opt, a game-theoretic approach for optimizing in-motion wireless charging efficiency, minimizing EVs' driving time to the charger, and avoiding traffic congestion at the charger lanes to fulfill this task. We studied a metropolitan-scale dataset of public transportation EVs and observed the EVs' spatial and temporal preference in selecting chargers, competition for chargers during busy charging times, the relationship between vehicle density and driving velocity on a road segment, the normal distribution of travel time of road segments, and the fact that vehicles have similar frequently driven trajectories. Based on the observations, a central controller estimates the vehicle density of the road segments by measuring the vehicles' trajectory travel time, the friendship among the vehicles, and the vehicles' routing choice given the presence of charger lanes. Then, we formulate a non-cooperative Stackelberg game between all the EVs and the central controller, in which each $\mathrm{EV}$ aims at minimizing its charging time cost to its selected target charger, while the central controller tries to maximally avoid the generation of congestion on the way through the in-motion wireless chargers. Our trace-driven experiments on SUMO demonstrate that WPT-Opt can maximally reduce the average charging time cost of the EVs by approximately $200 \%$ during different hours of a day.
\end{abstract}

\section{CCS Concepts: • Human-centered computing $\rightarrow$ Ubiquitous and mobile computing;}

Additional Key Words and Phrases: Wireless power transfer, EV driving velocity optimization, game theory

ACM Reference format:

Li Yan and Haiying Shen. 2021. Utilizing Game Theory to Optimize In-motion Wireless Charging Service Efficiency for Electric Vehicles. ACM Trans. Cyber-Phys. Syst. 5, 2, Article 19 (January 2021), 26 pages.

https://doi.org/10.1145/3430194

\section{INTRODUCTION}

Due to foreseen depletion of fossil fuels, many countries are actively adopting Electric Vehicles (EVs) for public transit systems [1]. The public service EVs (e.g., taxicabs and buses) are expected to keep driving without a long period of recharge downtime, although they only have limited

This research was supported in part by U.S. NSF grants ACI-1719397 and CNS-1733596 and Microsoft Research Faculty Fellowship 8300751.

Authors' addresses: L. Yan, Massachusetts Institute of Technology and University of Virginia; email: liyan_20@mit.edu; H. Shen, University of Virginia; email: hs6ms@virginia.edu.

Permission to make digital or hard copies of all or part of this work for personal or classroom use is granted without fee provided that copies are not made or distributed for profit or commercial advantage and that copies bear this notice and the full citation on the first page. Copyrights for components of this work owned by others than ACM must be honored. Abstracting with credit is permitted. To copy otherwise, or republish, to post on servers or to redistribute to lists, requires prior specific permission and/or a fee. Request permissions from permissions@acm.org.

(C) 2021 Association for Computing Machinery.

2378-962X/2021/01-ART19 \$15.00

https://doi.org/10.1145/3430194 
driving range (e.g., $200 \mathrm{~km}$ ) due to battery capacity. Wireless Power Transfer (WPT) techniques for in-motion EV charging $[2,3]$ and the deployment of in-motion wireless chargers (wireless chargers in short) [4] provide a solution to the above expectation. A road segment equipped with a wireless charger is called a charger lane. However, an EV may suffer from traffic congestion (i.e., long driving time) on the way to its selected target charger or long non-charging time at the charger without recharge (non-charging time in short). EV charging scheduling system should ensure the chargers' service efficiency (i.e., as many charged EVs as possible per unit time and each passed EV can be fully charged) [5].

Many scheduling strategies of EVs for improving the service efficiency of plug-in charging stations have been proposed [5-12]. Generally, they recommend target charging stations, which result in the minimum charging time cost (i.e., charger seeking time before reaching their target charging station plus non-charging (waiting) time at the charging station), to EVs based on current status of charging stations (e.g., location, number of available chargers). However, their charging time cost is estimated based on current traffic status. If the availability (i.e., number of available chargers) of the target charging station or the traffic on the road segments to the station changes, then the EVs may suffer from traffic congestion and a long charging time cost. What's more, these methods are not applicable for in-motion wireless chargers due to different charging approaches. For example, before busy charging times, the chargers may be non-congested. However, if legions of EVs drive to the currently "optimal recommended wireless charger lane" (i.e., the wireless charger lane with the shortest estimated charging time cost), then they may crowd into the wireless charger lane or the road segments connecting the chargers simultaneously. Such competition for the wireless charger lane may result in traffic congestion, which will greatly deteriorate the service efficiency of the charger lane.

However, the solution is non-trivial. Most charger deployment methods (including plug-in and in-motion wireless chargers) [4,13-15] advocate deploying plug-in chargers to the positions with high volume of EV traffic to offer easy access for EVs. However, the chargers deployed at these positions may frequently suffer from traffic congestion. Since congestion will greatly decrease EVs' passing velocity at the wireless charger lane [2], it will result in fewer EVs passing through the charger lane during a unit time (i.e., degraded charger service efficiency). What is worse, traffic congestion may also cause the drivers to waste much time before receiving a recharge. Thus, city planners always expect to avoid traffic congestion in their road networks, especially for road networks with a large EV population $[2,5]$. The road congestion on a wireless charger lane is measured by its vehicle density (i.e., number of vehicles per unit length); a higher vehicle density increases the service efficiency of the charger lane but generates congestion and decreases vehicle velocity, and vice versa. Therefore, it is a challenge to maximize the service efficiency of a network of wireless chargers while proactively avoiding the generation of congestion at the chargers and on the road segments to them.

To tackle the challenge, we propose WPT-Opt, a game-theoretic approach for optimizing inmotion wireless charging service efficiency. We first analyzed a metropolitan-scale vehicle mobility dataset that records the trajectories of 15,610 taxicabs, among which 6,510 vehicles are EVs, and 12,386 vehicles are electric customized transit service vehicles. A customized transit service vehicle is a local transit vehicle that picks up passengers on demand and its service is similar as that of Uber and Lyft. Our analysis confirms the competition among the EVs for popular charging stations, the normal distribution of EVs' travel time on road segments, and the fact that vehicles have similar frequently driven trajectories. Based on these observations, we first design a method that utilizes the historical trajectories and the friendship among the EVs to predict the future vehicle density of the road segments. Then, we focus on a charging system consisting of two parts: a central controller (e.g., hosted in cloud or fog) as the service provider, which outputs charging service information 
to the EVs and All EVs as the service follower. We formulate a non-cooperative Stackelberg game, in which each EV aims at minimizing the charging time cost to its target charger lane, while the central controller tries to maximize the service efficiency of all the wireless chargers. That is, each EV always wants to drive by its expected fastest velocity but may neglect the potential risk of traffic congestion, which conflicts with the the central controller, which wants to maximize the service efficiency of the wireless chargers and vehicle flow rate (i.e., average number of vehicles per unit time) of the road segments to them. After the Stackelberg equilibrium is reached, when the EVs follow their optimal velocities (i.e., fastest velocity without causing traffic congestion), the service efficiency of the wireless chargers and the vehicle flow rate of the road segments to them are maximized. In summary, our contributions include the following:

1. Our analysis on a metropolitan-scale EV mobility dataset confirms the movement and charging characteristics of EVs, the normal distribution of travel time of road segments, and the fact that vehicles have similar frequently driven trajectories, and lays the foundation for the design of WPT-Opt.

2. We propose a wireless charging service efficiency optimization strategy that utilizes a non-cooperative Stackelberg game between the central controller and all EVs to minimize each EV's charging time cost and meanwhile maximizes the service efficiency of wireless chargers.

3. We have conducted extensive trace-driven experiments to show the effectiveness of WPTOpt in terms of the number of charged EVs per unit time, the charging time cost of the EVs, and vehicle density on the wireless chargers. Compared with previous methods, WPT-Opt can maximally reduce the average charging time cost of the EVs by approximately $200 \%$ during different hours of a day.

To our knowledge, this article is the first work on optimizing the service efficiency of wireless chargers from the perspective of avoiding EV traffic congestion in the future. The remainder of the article is organized as follows. Section 2 presents a literature review. Section 4 presents our dataset analysis results. Section 5 presents the detailed design of WPT-Opt. Section 6 presents performance evaluations. Section 7 concludes the article with remarks on future work.

\section{RELATED WORK}

EV scheduling methods. Ma et al. [6] proposed to schedule EVs to under-utilized plug-in charging stations with reduced charging price to meet the respective charging demand of each EV and avoid extreme charging load on the power grid. Gan et al. [7] further predict the peak electricity-using hours of EVs at charging stations and reduce the price of recharging for each EV to avoid extreme charging load. Sundstrom et al. [12] proposed to minimize the cost of electricity and overload on the power grid through personalizing a charging plan (i.e., when and where to charge) for each EV. Kim et al. [11] proposed to rank EVs' charging requests by arrival time and estimated charging delay, and schedule the EVs to charging stations by their ranks to reduce the EVs' non-charging time. Qin et al. [10] and Lu et al. [9] considered the remaining power of EVs and the number of available chargers in charging stations to minimize the EVs' non-charging time. Malandrino et al. [8] modeled EVs' charging behavior (e.g., where and when to charge) and availability of charging stations with game theory to find the optimal charging price of each charging station that balances the charging load. Tian et al. [5] proposed to use each EV's historical recharging events, real-time trajectories and current traffic state to recommend the EV a charging station that leads to the minimal charging time cost. However, these methods are not directly applicable for wireless chargers, because they cannot avoid the generation of traffic 
congestion on the recommended charger lane or on the road segments to the charger lane in the near future, which may severely degrade the service efficiency of a charger lane.

Vehicle future mobility based routing. Wu et al. [16] found the spatio-temporal correlation in vehicle mobility and noted that the future trajectory of a vehicle is correlated with its past trajectory. In Trajectory-based Data Forwarding Scheme [17], Trajectory-based Statistical Forwarding Scheme [18], and Shared-Trajectory-based Data Forwarding Scheme [19], trajectory information of vehicles is collected through access points and used to predict vehicle mobility for data forwarding. Our work is based on the observations that trajectories illustrate vehicles' future mobility, which can be used to estimate future road vehicle density.

\section{BACKGROUND AND MOTIVATION}

\subsection{Definitions and Preliminaries}

A road network is a directed graph, in which vertices represent landmarks (i.e., intersections or turning points), and edges represent road segments connecting the landmarks [20]. We have the following definition for a vehicle trajectory.

Definition 3.1 (Trajectory). $V_{i}$ 's trajectory consists of the a start position $P_{i}^{s}$, an end position $P_{i}^{e}$, and a sequence of time-ordered landmarks, $\Phi_{i}^{l}:\left\{P_{i}^{s},\left(p_{0}, t_{0}\right), \ldots,\left(p_{j}, t_{j}\right), \ldots,\left(p_{N_{i}^{l}-1}, t_{N_{i}^{l}-1}\right), P_{i}^{e}\right\}$, where $p_{j}$ is a landmark's GPS position. $N_{i}^{l}$ is the total number of landmarks covered by this trajectory.

A vehicle's movement record is continuous. As in Reference [20], if a vehicle has stayed at a stop position for a long period of time (e.g., 10 minutes), then we determine that the vehicle has finished its previous trajectory. Thus, such stop positions cut the vehicle's continuous movement into several trajectories.

\subsection{Vehicle Flow Rate and Velocity at Chargers Matters}

Vehicle density of a road segment $s_{i}$ (denoted by $d_{i}$ ) is defined as the average number of vehicles per mile in the road segment (veh/mile), and the vehicle flow rate of $s_{i}$ (denoted by $r_{i}$ ) is defined as the average number of vehicles driving through $s_{i}$ per unit time $[20,21]$. That is, the vehicle flow rate of $s_{i}$ equals to the product of vehicle density and average vehicle passing velocity on $s_{i}$ (denoted by $\left.v_{i}\right): r_{i}=d_{i} \cdot v_{i}$. Moreover, the amount of energy transferred to an EV from a wireless charger lane is dependent on the EV's passing velocity [2,3]. Each wireless charger lane has a specified $\mathrm{EV}$ passing velocity $v_{i}^{\prime}$. An EV will be fully charged only when it drives through the charger lane with a velocity equal to or lower than $v_{i}^{\prime}$. Also, since different EVs have different battery capacities, their full recharge time for the same charger lane will be different. To ensure that all the EVs can be fully charged, we use the maximum battery capacity of the EVs to determine the $v_{i}^{\prime}$ of each charger lane. Therefore, we can see that to maximize the service efficiency of a wireless charger lane, we need to increase the vehicle flow rate at the charger lane and the road segments to it as much as possible, and meanwhile ensure that the EVs will pass the charger lane with the charger's specified velocity.

\section{METROPOLITAN-SCALE DATASET MEASUREMENT}

\subsection{Dataset Description and Data Processing System}

Our datasets are collected from Shenzhen, China $(1.1-12.31,2015)$, with a recording period of $30 \mathrm{~s}$ :

1. Taxicab Dataset. It is collected by the Shenzhen Transport Committee, which records the status (e.g., timestamp, position, velocity, SoC status) of 15,610 taxicabs, among which 6,510 of them are EVs. 
2. Dada Car Dataset. It is provided by the Dada Car corporation (a customized transit service similar to UberPool), which records the status (e.g., timestamp, position, velocity) of 12,386 electric reserved service vehicles.

3. Road Map. The road map of Shenzhen is obtained from OpenStreetMap [22]. According to the municipal information of Shenzhen [1], we use a bounding box with coordinate $($ lat $=22.4450$, lon $=113.7130)$ as the south-west corner, and coordinate (lat $=$ 22.8844 , lon $=114.5270)$ as the north-east corner, which covers an area of around $2,926 \mathrm{~km}^{2}$, to crop the road map data.

4. Charging Station Dataset. It is also collected by the Shenzhen Transport Committee, which records the information (e.g., GPS position, number of chargers) of 81 existing plugin charging stations in Shenzhen. The number of chargers in the charging stations ranges from 4 to 28. The charging stations are open to all EVs.

In the data analysis, we only selected the movement records of electric taxicabs and Dada cars for data analysis. We directly use the method introduced in Reference [5] to determine whether an EV is approaching its target charging station, recharging at the station or leaving the station. Specifically, if an EV's movement record shows that it has stayed at a charging station for a long period of time (e.g., 10 minutes), then we consider that it was recharging at the station at that time. Therefore, the charger seeking time before reaching the EV's target charging station is defined as the time interval between the time that the EV decides to have a recharge and the time it enters the target charging station for a recharge; the non-charging time of the EV at the target charging station is defined as the time duration it stays at the charging station but is not receiving recharge. Although our dataset analysis is applied on plug-in charging stations, we believe that the collected results are representative under the case of wireless chargers, since the EVs' long-term pattern (e.g., frequently driven road segments, working hours) remains relatively stable. Due to the limit of privacy protection, we can only access the mobility records of public service vehicles such as taxicabs and Dada cars. However, according to previous works [23, 24], the dataset covers almost all the public service vehicles that have charging demands. Therefore, the dataset is sufficiently representative for extracting observations related to EVs' charging patterns in a metropolitanscale road network. Moreover, to make the charging service efficiency optimization strategy more adaptive to the various driving preferences of general-purpose vehicles (e.g., private cars), we also propose methodologies to take into account drivers' routing choice (Section 5.2.3) and velocity selection (Section 5.3).

\subsection{EVs' Spatial Preference on Chargers}

The charge count of a charging station is defined as the number of EVs that charged at this charging station. It was indicated that an EV may have its own spatial preference in selecting charging stations [5]. In this analysis, we attempt to verify if the difference in the preference of selecting charging stations is conspicuous among different EVs, and the charging popularity (i.e., daily average charge count of EVs) differs significantly among different charging stations.

We measured the number of charging stations an EV visited more than 1 time per day in average throughout 2015. Figure 1 shows the results. We can see that $80 \%$ of the EVs only charged at fewer than 10 charging stations per day in average. For the remaining $20 \%$, the maximum number of visited charging stations is only around 20. This means that EVs have quite stable preference in selecting charging stations. If many EVs charge at the same charging stations, then they may cause congestion at these charging stations or the road segments to them.

We further measured the total daily average charge count of all the charging stations. Figure 2 shows the Cumulative Density Function (CDF) of the results. We can see that only $20 \%$ of the 


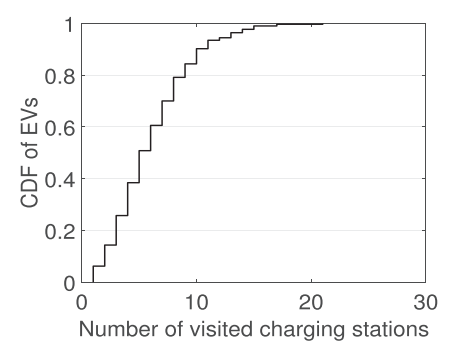

Fig. 1. Frequently visited charging stations of all vehicles.

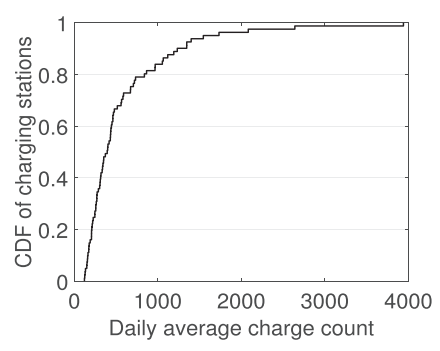

Fig. 2. Distribution of daily average charge counts of all charging stations.

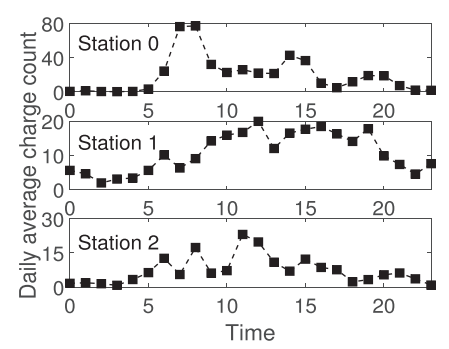

Fig. 3. Temporal preference on three charging stations.

charging stations have a daily average charge count higher than 1,000. But the highest value can be as high as around 4,000. The charging station with the highest value has 28 chargers. This result confirms that the charging stations have different levels of popularity among the EVs. The competition of the EVs for popular charging stations must be avoided to prevent the generation of possible congestion, which will result in long non-charging time of the EVs.

\subsection{EVs' Temporal Preference on Chargers}

The busy charging times (i.e., hours with relatively more charge counts) of the charging stations may also be quite different. To confirm this, we randomly selected three charging stations and measured the daily average charge count during each hour of a day throughout 2015. Figure 3 shows the measured results. We can see that for Station 0 (16 chargers), its busy charging time happens between 06:00 and 10:00; for Station 1 (10 chargers), its busy charging time happens between 08:00 and 20:00; and for Station 2 (19 chargers), its busy charging time happens between 10:00 and 14:00.

To explicitly illustrate the difference between the busy charging times of the charging stations, we further measured the maximum daily average charge count of each charging station and its corresponding hour. Figure 4 shows the measured results with a density scatter heat plot between the maximum number of charge count of each charging station and its corresponding time. Each point represents a charging station. The warmer color a point has, the more concentrated it is with other points, which have similar metric values. We can see that most busy charging times happen at around 09:00, 16:00, and 19:00 (i.e., points surrounded by red squares). These results illustrate that for different charging stations, their busy charging times are different, and their maximum charge counts are also quite different. To identify whether such charging counts will degrade the service of some charging stations, we further investigated the competition for chargers among EVs.

\subsection{Competition for Chargers Among EVs}

Previous methods schedule EVs based on current status of EV traffic and current availability of charging stations. If an EV's velocity is individually optimized without considering other EVs' future mobility, then many EVs may crowd into some charging stations or road segments to them and generate congestion. To illustrate this problem, we measured the daily average charge count and the average vehicle charging time cost to each of the 81 charging stations. The results are illustrated in Figure 5 with a density scatter heat plot. We can see that most charging stations (i.e., points with the warmest colors) have a relatively low daily average charge count (i.e., $<100$ ) but a relatively high vehicle charging time cost (i.e., $\approx 25$ minutes). This is because they are relatively distant to the areas that the EVs frequently visit, so the EVs need to drive a long time to reach 


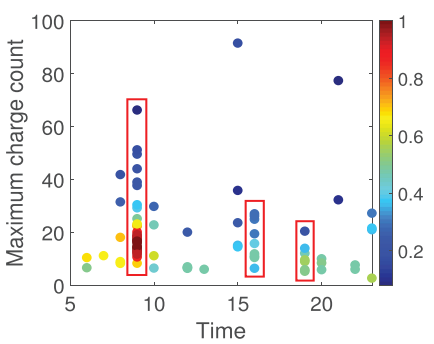

Fig. 4. Scatter plot of maximum charge count vs. corresponding time.

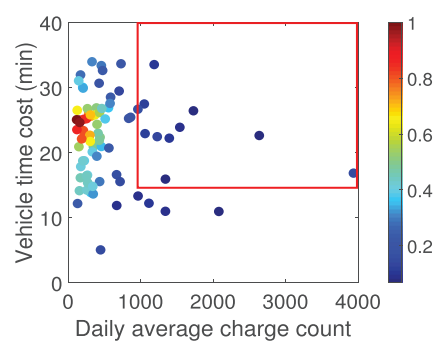

Fig. 5. Competition for charging stations.

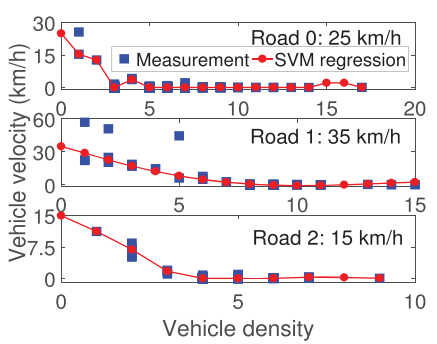

Fig. 6. Relation between vehicle density and actual driving velocity.

them. However, we still see that for a few charging stations marked by the red square, they are frequently visited by the EVs (i.e., >1000) but result in long charging time costs for the EVs (i.e., $>15$ minutes). This result confirms that EVs do have competition for certain charging stations.

\subsection{Relation between Vehicle Density and Driving Velocity on A Road Segment}

It has been indicated that vehicles' actual driving velocity on a road segment is subject to the vehicle density of the road segment [25]. However, previous work has demonstrated that the accurate relation between the actual vehicle driving velocity and the vehicle density of a road segment cannot be modeled with a parametric function (e.g., linear function) [26]. Support Vector Machine Regression (SVMR) is effective in learning the nonlinear relation between several variables [27]. For each road segment, we may use its historical records of vehicle density and vehicle passing velocity during a period (e.g., 15 minutes) to train the SVMR model and use the learned relation function to estimate the actual vehicle driving velocity given its vehicle density.

We randomly selected three road segments, of which velocity limits are $25 \mathrm{~km} / \mathrm{h}, 35 \mathrm{~km} / \mathrm{h}$, and $15 \mathrm{~km} / \mathrm{h}$, and measured the vehicle density and average vehicle passing velocity with a period of 15 minutes from July 1 to July 31 in 2015 . These road segments are potentially suitable for deploying wireless charger lanes according to previous charger lane deployment works [4] due to their relatively slow vehicle passing velocities. But such road segments are more prone to traffic congestion than others if without a proper traffic control mechanism due to their narrow road width and slow EV passing velocity. Then, for each road segment, we feed its measured results to the SVMR model to learn the relation function between its vehicle density and actual vehicle driving velocity. The results are illustrated in Figure 6. We can see that for the three road segments, the actual vehicle driving velocity generally decreases as the vehicle density increases, but with different decreasing rates. Also, the SVMR results fit the historical records with acceptable precision. In Section 5.2.4, we will elaborate how we use the estimated actual vehicle driving velocity to calculate the travel time of a vehicle trajectory and predict a road segment's future vehicle density.

\subsection{Distribution of Travel Time of Road Segments}

A vehicle trajectory is composed of several road segments. If we can estimate the travel time of each composing road segment with a sufficient accuracy, then we can determine the vehicle's position on the trajectory in the near future, which can be used to estimate the vehicle density of the road segment. Several previous works $[28,29]$ indicated that the travel time of a road segment can be generally described by normally distributed and statistically independent random variables with an acceptable precision and have confirmed their assumption with the vehicle travel time data collected from the CityLink Tollway in Melbourne, Australia. To verify whether this observation 


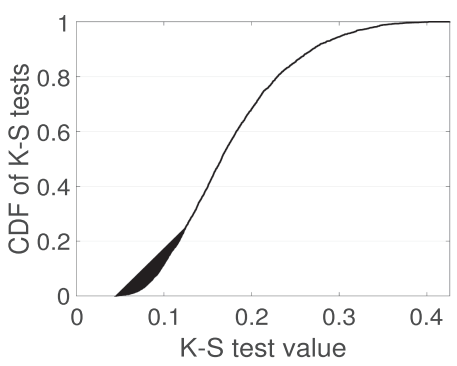

Fig. 7. K-S test values of all the passed tests.

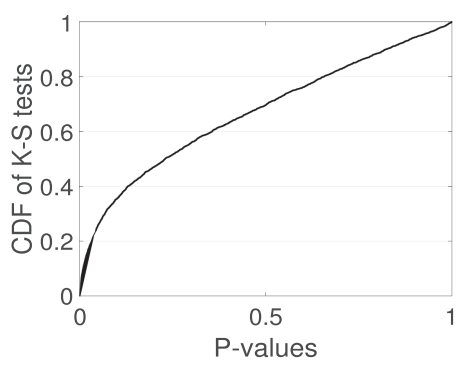

Fig. 8. The $p$ values of the passed tests.

also holds for metropolitan road networks, we collected all the historical travel time records of each road segment reflected in the Shenzhen trajectory dataset. First, we apply the One-sample Kolmogorov-Smirnov (K-S) test, which measures the maximum absolute difference between the population CDF of the actual data and the hypothesized CDF [30], on the historical travel time records of each road segment to identify whether the travel time of each road segment can be described with the normal distribution with a sufficiently high accuracy. Specifically, for a road segment, the K-S test value between the population CDF of its actual travel time (denoted as $F(t)$ ) and the hypothesized CDF (denoted as $\left.\hat{F}_{h}(t)\right)$ is calculated by the following:

$$
K=\max _{t}\left(\left|F(t)-\hat{F}_{h}(t)\right|\right),
$$

where $K$ is the K-S test value and $\max _{t}$ is the maximum absolute difference between $F(t)$ and $\hat{F}_{h}(t)$. Then we use $K$ to calculate the significance level (measures the reliability of this test) by using an approximation formula or by referring to the interpolation table as in Reference [30]. If the test approves the hypothesis that $F(t)$ is similar to $\hat{F}_{h}(t)$ (i.e., a small K-S test value) at the significance level of $5 \%$, then we view the observed travel time records follow the normal distribution. Finally, we count the number of road segments that pass the hypothesis test and calculate the total pass rate over all the road segments. The test pass rate of all the road segments is $85.6 \%$. Figure 7 shows the CDF of the K-S test values of all the passed tests. We can see that more than $80 \%$ of the passed tests have a K-S test value lower than 0.25 , which means the maximum absolute difference between the statistical distribution of the actual data and the statistical distribution of the hypothesized normal distribution is quite small.

To further measure the confidence of reliability of the K-S test results, we further measured the $p$ values of the test results. The $p$ value represents the probability that the historical travel time records actually follow the hypothesized normal distribution. That is, the doubt on the validity of the test result. The smaller the $p$ value a K-S test has, the less reliable the K-S test result is, even if the historical travel time records have passed the K-S test [30]. Figure 8 shows the $p$ values of all the passed tests. We can see that around $80 \%$ of the passed tests resulted in a $p$ value higher than 0.1 . This means most of the test results are reliable. Therefore, the actual distribution of the travel time of most road segments follows the hypothesized normal distribution.

\subsection{Vehicles Have Similar Frequently Driven Trajectories}

4.7.1 Existence of Vehicle Routines. Several previous works [31,32] have confirmed that most vehicles in a city are prone to drive the same trajectories during certain time periods each day. For example, Jack usually drives from home to working place at around 08:00 via University Avenue and leaves from working place for home at around 18:00 via Campus Drive on weekdays. In this article, we define that if the ratio of a trajectory among all the trajectories of a vehicle during an 


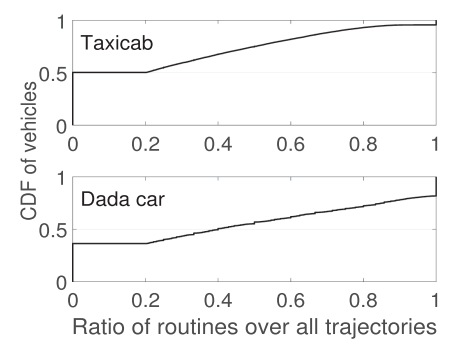

Fig. 9. Existence of routines in the trajectories of vehicles.

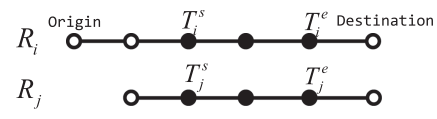

Fig. 10. Spatial similarity between $R_{i}$ and $R_{j}$.

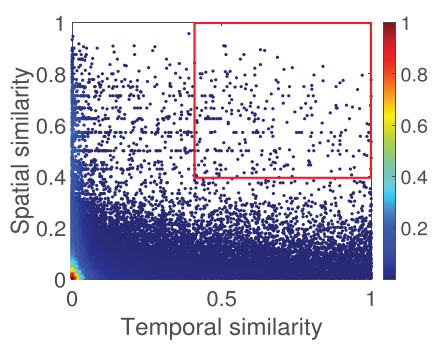

Fig. 11. Density scatter plot of temporal and spatial similarities of trajectories.

observation window, say, $N_{d}=30$ days, is higher than the routine ratio threshold, say, $\kappa=20 \%$, and the trajectory is driven at around a specific time (the standard deviation of the start times is no higher than a threshold, say, $\sigma_{s}=30$ minutes), then we view this trajectory as a routine of the vehicle. To confirm the existence of routines in the driving of vehicles, we identified the routines of each vehicle and calculated the ratio of routines over all of the vehicle's historical trajectories. Specifically, the CDF of the measured results of taxicabs and Dada cars are illustrated in Figure 9. We can see that for about $50 \%$ of the taxicabs, more than $20 \%$ of their driven trajectories are actually their routines, while for more than $60 \%$ of the Dada cars, more than $20 \%$ of their driven trajectories are their routines. The reason that Dada cars drive routines more often is that the routes reserved by Dada car passengers usually connect their homes and working places. Thus, many Dada cars frequently repeated the passengers' daily routines in their driving. These results confirm that for many vehicles, they do have routines in driving.

4.7.2 Identification of Similar Vehicle Routines. During certain time periods (e.g., rush hours), some vehicles may share common routines. For example, Jack and his neighbor, Mark, usually drive the same routine routes to commute from their home to their working place at around 08:30-09:00 every morning. Thus, during certain time periods, we may use the similarity of the routines of different vehicles to help estimate the vehicle density of the road segments covered by the routine routes. To identify whether two routines (say, $R_{i}$ of vehicle $V_{i}$ and $R_{j}$ of vehicle $V_{j}$ ) are similar, we use the longest sequence of common consecutive landmarks between $R_{i}$ and $R_{j}$ to measure their spatial similarity and their difference in the start time of the sequence of common landmarks as their temporal similarity.

Spatial similarity. To measure the spatial similarity between $R_{i}$ and $R_{j}$, we need to determine their maximum number of consecutive common landmarks. For example, in Figure 10, there are maximally three consecutive common landmarks (black dots) between $R_{i}$ and $R_{j}$. Finding the maximum number of consecutive common landmarks between $R_{i}$ and $R_{j}$ can be simplified to the problem of finding the Longest Common Substring (LCS) between the two strings of landmarks [33]. We use the standard dynamic programming [34] to obtain the LCS. Generally, while iterating all the substrings of $R_{i}$, we check whether any substring of $R_{i}$ also appears in $R_{j}$. After all iterations, we obtain the LCS between $R_{i}$ and $R_{j}$. Finally, we define the spatial similarity between $R_{i}$ and $R_{j}$ (denoted as $\gamma_{i j}$ ) as:

$$
\gamma_{i j}=\min \left\{\frac{|L C S|}{\left|R_{i}\right|}, \frac{|L C S|}{\left|R_{j}\right|}\right\},
$$

where $|\cdot|$ represents the number of landmarks in the sequence. The range of $\gamma_{i j}$ is $[0,1]$. The higher $\gamma_{i j}$ that $R_{i}$ and $R_{j}$ have, the more spatially similar they are. 
Temporal similarity. Even if there are common landmarks between two routines, their respective start times and end times of the common landmarks are unlikely to be the same. Also note that the start times and end times of the common landmarks of the two routines follow their respective distributions. Recall that the Two-sample K-S test measures whether the statistical distributions of two data samples are similar by comparing their CDFs [30]. Therefore, we use the Two-sample K-S test to determine whether both the difference between the distributions of the LCS start times and the difference between the distributions of the LCS end times in the two routines are small enough. As shown in Figure 10, suppose the start times of the LCS on $R_{i}$ and $R_{j}$ are $T_{i}^{s}$ and $T_{j}^{s}$, respectively, and the end times of the LCS on $R_{i}$ and $R_{j}$ are $T_{i}^{e}$ and $T_{j}^{e}$, respectively. We define the temporal similarity between $R_{i}$ and $R_{j}$ (denoted as $\tau_{i j}$ ) as:

$$
\tau_{i j}=\left\{\begin{array}{ll}
\min \left\{\eta_{i j}^{s}, \eta_{i j}^{e}\right\}, & \text { both } T_{i}^{s}, T_{j}^{s} \text { and } T_{i}^{e}, T_{j}^{e} \text { pass the K-S test } \\
0, & \text { otherwise }
\end{array} .\right.
$$

where $\eta_{i j}^{s}$ and $\eta_{i j}^{e}$ are the $p$ values of the Two-sample K-S test results on $T_{i}^{s}, T_{j}^{s}$ and $T_{i}^{e}, T_{j}^{e}$, respectively. The range of $\tau_{i j}$ is $[0,1]$. The higher $\tau_{i j}$ that $R_{i}$ and $R_{j}$ have, the more temporally similar they are.

Next, we measured the spatial and temporal similarities of every two routines from two different vehicles, respectively. We illustrate the measured results with a density scatter plot of the temporal similarity with respect to the spatial similarity as in Figure 11. Each point represents a measured result resulted from the comparison of the two routines. The density of the distribution of the measured results are demonstrated with color heat. The warmer color the points are represented, the more concentrated the comparison results distribute on the plot with corresponding spatial and temporal similarities. Since we are interested in identifying similar routines among different vehicles, we focus on the comparison results with both high spatial similarity (0.4) and high temporal similarity (0.4). We can see that such comparison results only take up a small portion within the red square circle. This means that some vehicles do have highly similar routines. During the occurrence time periods of the similar routines, these vehicles are likely to simultaneously drive on the same road segments. Thus, we propose to define the vehicles' friendship in terms of the spatial and temporal similarities of their routines. Then, given the presence of a vehicle, we can use the vehicle's friendship to estimate the presence probability of its friend vehicles' on the same road segment, which can further help us estimate the vehicle density of each road segment. The details of measuring the friendship among different vehicles and the utilization of vehicles' friendship in calculating road vehicle density are introduced in Section 5.2.2 and Section 5.2.4, respectively.

\section{SYSTEM DESIGN DETAILS}

\subsection{System Overview}

WPT-Opt consists of two parts: All EVs as the service follower and a central controller (e.g., hosted in cloud or fog) as the service provider, which outputs charging service information to the EVs. The system structure is shown in Figure 12. Above all, we have the following assumptions:

1. Each EV that has a charging request will first use previous methods [5-12] to determine its target charger lane. Meanwhile, it is willing to report and adjust its driving status (current position, velocity) according to the charging service information.

2. Non-electric vehicles and EVs without a charging request are also willing to report their current driving trajectory to the central controller for vehicle density estimation. This is reasonable, because these vehicles can receive better routing benefit from providing such information. 


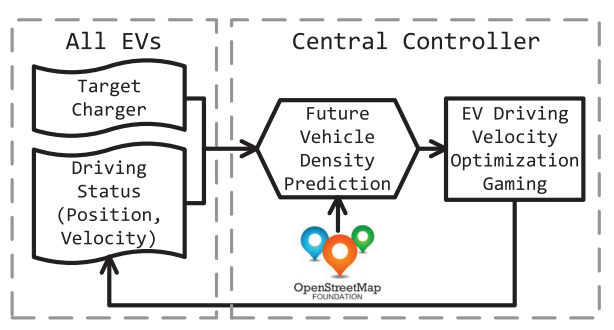

Fig. 12. System structure.

In Section 4.2 and Section 4.3, we have demonstrated that all EVs have their respective spatial and temporal preference on selecting charging stations, and such preferences can cause competition. To let all EVs reach their target charger lane as fast as possible, and meanwhile maximize the service efficiency of wireless chargers without generating congestion, we use the Stackelberg game [21] between the EVs and the central controller to determine the expected vehicle density that maximizes the service efficiency of the wireless chargers and the optimal driving velocity for each EV. Specifically, the EVs report its driving status (current position, velocity) to the central controller periodically (e.g., every 5 minutes). In response, the central controller outputs the optimized driving velocity for each EV periodically. At the start of current time slot $\mathcal{T}_{c}$ (e.g., 5 minutes), the central controller applies the future vehicle density prediction (Section 5.2) on the road segment set (i.e., the set of road segments connecting the positions of the EVs with a charging request and the EVs' target chargers) for the next time slot $\mathcal{T}_{c+1}$ :

1. Each EV keeps reporting its driving status (current position, velocity) and target charger lane to the central controller.

2. Based on the information collected from the EV, the central controller calculates its trajectory travel time in $\mathcal{T}_{c+1}$ to know the EV's possible position in any time during $\mathcal{T}_{c+1}$. Then the central controller aggregates the trajectories and predicts the vehicle density of each road segment in $\mathcal{T}_{c+1}$.

By the end of current time slot $\mathcal{T}_{c}$, an EV driving velocity optimization gaming (Section 5.3) is conducted between the central controller and all the EVs. The gaming process is executed periodically with a time interval $\mathcal{T}$ (e.g., 5 minutes):

1. Based on the predicted average vehicle density over all the road segments in road segment set in $\mathcal{T}_{c+1}$, the central controller determines a set of candidate expected average vehicle densities over the next time slot for the road segment set, which are achievable by vehicle velocity adjustment.

2. Based on each expected average density, each EV determines its actual driving velocity on its current road segment and reports it to the central controller.

3. The central controller determines the final expected average density that maximizes the service efficiency of wireless chargers and the vehicle flow rate of the road segments connecting the chargers, and notifies all the EVs.

4. Each EV chooses its velocity corresponding to the final expected average density.

We first explain how the central controller predicts the vehicle density of road segments (Section 5.2), and then present the non-cooperative Stackelberg gaming (Section 5.3). 


\subsection{Future Vehicle Density Prediction}

To make the gaming process work after each time slot $\mathcal{T}$ (e.g., 5 minutes), we must be able to calculate the future vehicle density of wireless chargers and the road segments to them. To this end, we must solve the following problems:

1. How to estimate the travel time to each road segment of an EV's future trajectory based on the current vehicle density of each road segment (i.e., $\mathcal{T}_{c}$ )? (Section 5.2.1)

2. How to utilize the future trajectories and the travel times to the road segments of the trajectories of all the EVs to predict the future vehicle density of each road segment in the next time slot (i.e., $\left.\mathcal{T}_{c+1}\right)$ ? (Section 5.2.4)

5.2.1 Trajectory Travel Time Calculation. With the current position and target charger lane periodically reported by each EV, the central controller uses an existing routing method (e.g., Reference [5]) to determine the EV's trajectory in the next time slot $\mathcal{T}_{c+1}$, which is a sequence of road segments connecting the EV's current position and target charger lane. Note that other nonelectric vehicles and EVs without a charging request also report their current driving trajectories to the central controller. It then utilizes the combination of all the trajectories to calculate the travel time of each road segment that will be passed in $\mathcal{T}_{c+1}$ by the EV. In our gaming process, we will determine a vehicle's actual driving velocity $\left(v_{i}\right)$ on a road segment $\left(s_{i}\right)$. Then, for each $s_{i}$, the estimated travel time on $s_{i}$ (denoted by $\widetilde{t_{i}}$ ) is $\widetilde{t_{i}}=l_{i} / v_{i}$, where $l_{i}$ is the length of $s_{i}$.

In Section 4.5, we have shown that the actual vehicle driving velocity $v_{i}$ of a road segment $s_{i}$ is related to its vehicle density $d_{i}$, and the relation is relatively stable but non-parametric, which is denoted as $v_{i}=f_{i}\left(d_{i}\right)$. SVMR model is effective in estimating the non-parametric function between two variables [27]. Thus, for each road segment, we build its SVMR model to learn its relation between $v_{i}$ and $d_{i}$. Specifically, we input its vehicle density as the predictor value and the corresponding actual vehicle driving velocity as the response value to the SVMR model. The output is the estimated relation function between the vehicle density and the actual vehicle driving velocity of the road segment. Later, given an estimated vehicle density of the road segment, we can use the function to output its actual vehicle driving velocity, and further estimate the travel time of the road segment.

Several previous works $[28,29]$ have confirmed that the travel time of a road segment can be described by normally distributed and statistically independent random variables with an acceptable precision. Therefore, for an EV, we estimate its travel time of the trajectory from its current position to $s_{i}$ as the sum of the travel times of the road segments included in the trajectory, $\widetilde{T}_{i}=\sum_{k=1}^{M_{i}} \widetilde{t}_{k}$, where $M_{i}$ is the number of the road segments included in the trajectory. Based on the historical records of the travel time of road segment $s_{k}$ from all vehicles, the central controller can calculate the variance of $s_{k}$ 's travel time $\sigma_{k}^{2}$ for each composing road segment. Then, the standard deviation of $\widetilde{T}_{i}$ is calculated by summing the variances of the composing road segments, $\Delta_{i}^{2}=\sum_{k=1}^{M_{i}} \sigma_{k}^{2}$. This is because $\widetilde{t}_{k}$ follows normal distribution. Finally, an EV's trajectory can be represented as a sequence of road segments it will pass in $\mathcal{T}_{c+1}$ and their corresponding estimated travel times $\left\{\left(s_{i}, \widetilde{T}_{i}\right) \mid i=1,2, \ldots, M\right\}$, where $M$ denotes the total number of road segments that the EV will pass in $\mathcal{T}_{c+1}$.

5.2.2 Measuring Friendship among Vehicles. Recall that the routines of a vehicle are defined as the trajectories frequently driven by the vehicle during specific time periods (Section 4.7). Based on this definition, we have developed the method for calculating the spatial similarity and temporal similarity of routines in Section 4.7.2. In Figure 11, we have also demonstrated that some vehicles do have highly similar routines (both spatial similarity and temporal similarity are higher than 0.4 ), 


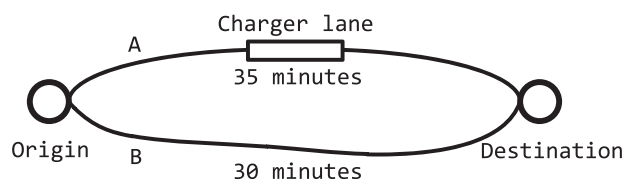

Fig. 13. EV drivers' routing choice behavior.

which means that the vehicles are likely to simultaneously drive on the same road segments during specific time periods. Driven by these observations, we define two vehicles have friendship only if they have at least one pair of routines, of which both spatial similarity and temporal similarity are higher than the similarity threshold, say, $\theta=0.4$. Specifically, given two routines, say, $R_{i}$ of vehicle $V_{i}$ and $R_{j}$ of vehicle $V_{j}$, the friendship strength between $V_{i}$ and $V_{j}$ reflected by $R_{i}$ and $R_{j}$ (denoted as $\left.\beta_{i j}\right)$ is calculated as the product of the spatial similarity $\left(\gamma_{i j}\right)$ and the temporal similarity $\left(\tau_{i j}\right)$ of the routines:

$$
\beta_{i j}=\left\{\begin{array}{ll}
\gamma_{i j} \cdot \tau_{i j}, & \gamma_{i j}>\theta \text { and } \tau_{i j}>\theta \\
0, & \text { otherwise }
\end{array} .\right.
$$

Note that the setting of the similarity threshold controls the tradeoff between the friendship coverage and friendship estimation accuracy. For example, for vehicle $V_{i}$, if the similarity threshold is relatively smaller, more vehicles will be viewed as friend vehicles of $V_{i}$, but the friendship is less reliable and may decrease the accuracy of road vehicle density calculation (Section 5.2.4), and vice versa. Thus, the value of the similarity threshold should be set according to different city road networks' requirement on the estimation of road vehicle density. The problem of utilizing the spatial and temporal similarities of the movement of nodes to increase the success rate of packet delivery has been studied in many papers focusing on Delay Tolerant Networks (DTN) [35-38]. For example, in our previous works: MobiT, which is designed for packet routing in Vehicular Delay Tolerant Networks [37], and TSearch, which is designed for node searching in DTNs [38], we use the friendship among different nodes to increase the estimation accuracy of the nodes' appearance position. However, these works do not consider the consecutive matching of positions covered in the trajectories and statistical overlapping of the start times, which are important for the prediction of vehicle traffic density. Thus, they are not directly applicable for solving the research problem of this article.

5.2.3 Queuing Theory-based Driver Routing Model. Charger presence might affect EV drivers' routing in a way that they are more likely to drive routes equipped with chargers to mitigate range anxiety $[39,40]$. For example, as shown in Figure 13, there are two candidate routes with approximate distances between the origin and destination. Due to the well-known range anxiety of EVs [41-43], the EV driver might choose route A, which has a charger lane, even if its travel time cost ( 35 minutes) is a bit longer than that of route B (30 minutes). There are primarily two factors that affect the EV driver's decision of choosing whether to charge on the way or drive directly to the destination: the travel time cost and the benefit brought by the charger lane. Therefore, the charger lanes may affect the traffic distribution on the road segments due to the impact of charger lane positions on traffic flow. It is necessary to consider this impact in calculating the vehicle density of the road segments.

According to References [39,44], the travel time cost of a candidate route $u$ consists of the driving time of normal road segments $\left(t_{u}^{d}\right)$, the passing time of charger lanes $\left(t_{u}^{c}\right)$ and the waiting time at the charger lanes $\left(t_{u}^{p}\right)$. The driving time of road segments included in the route $u$ can be 
calculated as:

$$
t_{u}^{d}=\sum_{n=0}^{N_{u}^{R}-1} \frac{l_{n}}{v_{n}}
$$

where $N_{u}^{R}$ is the number of road segments of the route $u$ and $v_{n}$ and $l_{n}$ are the actual vehicle driving velocity and length of the $n$th road segment, respectively.

The driving time of charger lanes consists of the EVs' waiting time before charging and charging time. Let $\lambda_{i}$ denote the arrival rate of EVs at the charger lane located at landmark $p_{i}$ (i.e., the number of EVs arriving at $p_{i}$ for charging per unit time), which is actually the vehicle flow rate of $p_{i}$. Let $\mu_{i}$ denote the service rate of the charger lane located at landmark $p_{i}$ (i.e., the number of EVs that the charger lane can charge per unit time), which is calculated as $\mu_{i}=v_{i} / L_{i}[2,45]$, where $v_{i}$ is the actual vehicle driving velocity at $p_{i}$ and $L_{i}$ is the charger lane length. Thus, an EV's charging time at the charger lane is

$$
t_{u}^{c}=1 / \mu_{i}
$$

The utilization ratio of the charger lane is $\rho_{i}=\lambda_{i} / \mu_{i}$. According to the $\mathrm{M} / \mathrm{M} / 1$ queuing theory $[39,46]$, the EVs' waiting time at the charger lane is

$$
t_{u}^{p}=\left\{\begin{array}{ll}
\frac{\rho_{i} / \mu_{i}}{1-\rho_{i}}, & \text { if } \rho_{i}<1 \\
\rho_{i}, & \text { otherwise }
\end{array} .\right.
$$

Finally, the travel time cost of the route $u$ between the Origin-Destination (O-D) pair $w$ can be calculated as

$$
T_{u}^{w}=t_{u}^{d}+x_{u}^{w}\left(t_{u}^{c}+t_{u}^{p}\right)
$$

where $x_{u}^{w}$ is the binary variable indicating the presence of charger lanes on the route $u$ and $x_{u}^{w}=1$ if there is at least one charger lane in $u$ and $x_{u}^{w}=0$ otherwise. Previous studies on EV drivers' routing behavior have confirmed that for an $\mathrm{EV}$ driver, the probability of choosing a candidate route can be described with a multinomial logit model [41-43]. Specifically, with the travel time cost and charger presence of a route $u$, the EV driver's probability of choosing $u$ among all the candidate routes between the O-D pair $w$ (denoted as $P_{u}^{w}$ ) can be estimated as

$$
P_{u}^{w}=\frac{\exp \left(\xi \frac{1}{T_{u}^{w}}+\varepsilon x_{u}^{w}\right)}{\sum_{k \in U^{w}} \exp \left(\xi \frac{1}{T_{k}^{w}}+\varepsilon x_{k}^{w}\right)}, \forall u \in U^{w}, w \in W,
$$

where $U^{w}$ is the set of all feasible routes of the O-D pair $w$ reflected in all the historical trajectory data; $W$ is the set of all possible O-D pairs on the road network; and $\xi$ and $\varepsilon$ are the scaling parameters for travel time cost and the presence of charger lanes, respectively, which describe the routing decision sensitivity in terms of travel time cost and the presence of charger lanes. In practice, $\xi$ and $\varepsilon$ should be calibrated by survey. In this study, we follow the settings of these parameters as recommended in Reference [41]: $\xi=0.1$ and $\varepsilon=0.8$. According to Equation (9), the longer travel time cost a route has, the lower probability an EV driver will choose the route and vice versa. This is consistent with the real-world driver's expectation of minimizing the travel time cost.

5.2.4 Road Vehicle Density Calculation. Due to the inaccuracy of the above estimation, the estimated travel time in $\left\{\left(s_{i}, \widetilde{T}_{i}\right) \mid i=1,2, \ldots, M\right\}$ has a certain probability to be accurate. That is, each vehicle only has a certain probability to appear on a road segment at the estimated travel time. Then, we use the probabilities of all the vehicles to calculate the vehicle density of each road segment in $\mathcal{T}_{c+1}$. Specifically, we first calculate the probability that a vehicle will appear at each road segment in its trajectory in $\mathcal{T}_{c+1}$. Then, we sum up all the vehicles' appearance probabilities at a road segment in $\mathcal{T}_{c+1}$ as the vehicle density of the road segment in $\mathcal{T}_{c+1}$. 
Given the next time slot $\mathcal{T}_{c+1}=\left[t_{j}^{s}, t_{j}^{e}\right]$ (e.g., [00:00,00:05]), where $j$ means it is the $j$ th time slot in a day, $t_{j}^{s}$ and $t_{j}^{e}$ are the start time and end time of the time slot, respectively. The central controller then measures each vehicle's appearance probability at $s_{i}$ during $\left[t_{j}^{s}, t_{j}^{e}\right]$ by referring to the vehicle's estimated travel time to $s_{i}$. Therefore, we can calculate the vehicle's appearance probability at $s_{i}$ during $\left[t_{j}^{s}, t_{j}^{e}\right]$ as

$$
P\left(T_{i} \leqslant t_{j}^{e}-t_{j}^{s}\right)=\Phi\left(\frac{t_{j}^{e}-t_{j}^{s}-\widetilde{T}_{i}}{\Delta_{i}}\right)-\Phi\left(\frac{-\widetilde{T}_{i}}{\Delta_{i}}\right),
$$

where $T_{i}$ denotes the EV's actual travel time from current position to $s_{i}$ and $\Phi(\cdot)$ is the CDF of the standard normal distribution with mean $\widetilde{T}_{i}$ and standard deviation $\Delta_{i}$. Based on the historical records of all vehicles' travel time on $s_{i}$, we can calculate the CDF of the travel time on $s_{i}$. By summing up the appearance probabilities of the vehicles on $s_{i}$ during $\mathcal{T}_{c+1}$, the central controller estimates the vehicle density of each $s_{i}$ in $\mathcal{T}_{c+1}$ as

$$
d_{c+1}^{s_{i}}=\sum_{k=1}^{N} P_{k}\left(T_{i} \leqslant t_{j}^{e}-t_{j}^{s}\right)+\sum_{k=1}^{N} \sum_{f=1}^{F_{k}} \beta_{k f} P_{f}\left(T_{i} \leqslant t_{j}^{e}-t_{j}^{s}\right)+\sum_{w \in W} \sum_{u \in U_{i}^{w}} P_{u}^{w},
$$

where $N$ is the number of vehicles that will pass $s_{i}$ during $\left[t_{j}^{s}, t_{j}^{e}\right]$ and $F_{k}$ is the number of friends of the $k$ th vehicle. Note that the $k$ th vehicle may have multiple pairs of similar routines with its friend vehicles, but the similar routines may cover different time periods. Wether the similar routine of a friend vehicle (say, the $f$ th friend vehicle) can help estimate the vehicle density of $s_{i}$ is dependent on the friend vehicle's appearance probability at $s_{i}$ during $\left[t_{j}^{s}, t_{j}^{e}\right]$, which is denoted as $P_{f}\left(T_{i} \leqslant t_{j}^{e}-t_{j}^{s}\right)$. Note that the friendship strength between the $k$ th vehicle and its $f$ th friend vehicle (i.e., $\beta_{k f}$ ) is primarily determined by the long-term observation window size of the two vehicles' routines $N_{d}$, the routine ratio threshold $\kappa$, the start time deviation threshold $\sigma_{s}$, and the similarity threshold $\theta$. For example, if the observation window is relatively shorter, then the computation overhead will be lower, but the determination of the vehicles' routines may be inaccurate due to the lack of historical trajectories. This may bring high uncertainty to the estimation of the friendship strength between the vehicles, because the inaccurate routines cannot fully capture the long-term movement patterns of the vehicles. Thus, the parameters related to the determination of vehicle friendship control the tradeoff between the coverage of the uncertainty and computation overhead, and should be set according to different city road networks' traffic characteristics. Recall that $W$ is the set of all possible O-D pairs on the road network, $U_{i}^{w}$ is the set of historical routes that pass through road segment $s_{i}$, and $P_{u}^{w}$ is the vehicles' probability of choosing the route $u$ among all the candidate routes between the O-D pair $w$. Thus, $\sum_{w \in W} \sum_{u \in U_{i}^{w}} P_{u}^{w}$ is the vehicle density contributed by the presence/non-presence of chargers on $s_{i}$.

\subsection{EV Driving Velocity Optimization Gaming}

5.3.1 Overview. We refer to a previous work on traffic optimization [47] for the establishment of the Stackelberg game. In the Stackelberg game, the service leader (i.e., central controller) considers the predicted average vehicle density of a road segment, and then chooses a set of expected vehicle densities, $D=\left\{d_{1}, d_{2}, \ldots, d_{n}\right\}$, that are achievable by vehicle velocity adjustment. The central controller hopes to evenly distribute the EVs over road segment set by properly assigning a $d$ value. The EV drivers receive $D$ from the central controller and picks a velocity in response to each $d_{i}$ to maximize its own utility (driving as fast and safely as possible while minimizing the risk of congestion). Next, the central controller selects the vehicle density, which is denoted by $d_{l}$, that maximizes the service efficiency of the wireless chargers and vehicle flow rate of the road 
segments connecting them, and then the EVs choose their velocities corresponding to the selected $d_{l}$. Finally, we solve the Stackelberg equilibrium of the game, i.e., the game reaches a state that the service efficiency of wireless chargers is maximized while the EV drivers are satisfied with the driving status (judged by driving velocity and associated risk of congestion). The gaming is executed periodically. In the following, we first introduce the utility of an EV driver and the utility of the central controller, and then introduce the gaming between them.

5.3.2 Utility Function of EV Drivers. For EV drivers, we define a utility function as the level of benefit an EV driver can obtain through driving by a certain velocity on road segment $s_{i}$. An EV driver can receive more benefit (i.e., arrive at its target charger lane earlier than expected) if it drives at a relatively higher velocity. However, as discussed in Section 1, if all EVs drive at their fastest velocities, their risk of suffering from congestion may increase. Therefore, we formulate an EV driver's utility function as a value calculated by subtracting the potential risk of congestion $\left(U_{r}(\cdot)\right)$ from the driver's satisfaction degree $\left(U_{s}(\cdot)\right)$ resulted from driving fast, as shown in Equation (12),

$$
\begin{aligned}
F\left(v_{i}, \alpha_{i}\right) & =U_{s}\left(v_{i}, \alpha_{i}\right)-U_{r}\left(d, v_{i}\right) \\
\text { s.t. } \quad v_{i} & \leqslant v_{i}^{\text {max }},
\end{aligned}
$$

where $v_{i}$ is the vehicle's velocity for optimization, which is selected by the vehicle itself; $\alpha_{i}$ is a scale factor to make $U_{s}(\cdot)$ and $U_{r}(\cdot)$ comparable.

Specifically, an EV driver's satisfaction degree $U_{s}(\cdot)$ is primarily determined by its driving velocity [21]. $U_{s}(\cdot)$ ought to be non-decreasing as each driver desires high velocity (i.e., short driving time to its target charger lane). Also, $U_{s}(\cdot)$ should reach the largest value only when the vehicle is driving at the velocity corresponding to the vehicle density expected by the central controller, which is denoted as $f_{i}(d)$. Meanwhile, the derivative of the satisfaction degree is non-increasing, because the driver's satisfaction degree gradually gets saturated when the vehicle velocity increases to some level [25]. Considering these properties, we design $U_{s}(\cdot)$ as a concave function. Since the Natural Logarithmic Functions are representative concave functions [48], we define

$$
U_{s}\left(v_{i}, \alpha_{i}\right)=\alpha_{i} \cdot \ln \left(v_{i}\right)
$$

An EV driver's potential risk of congestion is closely related to its vehicle flow rate [21]. As the $\mathrm{EV}$ is expected to drive by the velocity corresponding to the $d$ (i.e., $f_{i}(d)$ ), we use a Sigmoid function to approximate the probability of congestion with respect to the EV's selected driving velocity. If the EV drives above the threshold $f_{i}(d)$, then the probability of congestion increases significantly. Therefore, we formulate an EV driver's potential risk of congestion as

$$
U_{r}\left(d, v_{i}\right)=\frac{1}{1+e^{-\left(v_{i}-f_{i}(d)\right)}} d v_{i}
$$

Note that the value $d$ is determined by the central controller based on the estimated vehicle density of each road segment in the road network (determined by Equation (11)). Given that Equation (11) is affected by the future trajectories and friendship among all the vehicles, the potential risk of congestion is dependent on the random factors such as the movement of all the vehicles and the EV drivers' response to the central controller.

As the EV increases its velocity, its utility will first increase to the maxima at some velocity around $f_{i}(d)$, and then decrease. Thus, the EV's velocity is adjusted by the central controller and meanwhile can drive by a relatively fast velocity. Combining Equation (13) and Equation (14) into 
Equation (12), we have

$$
\begin{aligned}
F\left(v_{i}, \alpha_{i}\right) & =\alpha_{i} \cdot \ln \left(v_{i}\right)-\frac{1}{1+e^{-\left(v_{i}-f_{i}(d)\right)}} d v_{i} \\
\text { s.t. } \quad v_{i} & \leqslant v_{i}^{\max } .
\end{aligned}
$$

5.3.3 Utility Function of Central Controller. The central controller always aims at maximizing EV flow rate on wireless chargers and the road segments connecting them. Also, recall that each wireless charger lane has a specified EV passing velocity $v_{i}^{\prime}$, which enables the wireless charger lane to fully charge the EV after driving through the charger lane (Section 3.2). Correspondingly, the utility function of the central controller is defined as:

$$
L(d)=\sum_{i=1}^{N_{s}} d_{i} \cdot v_{i}+\sum_{i=1}^{N_{e}} d_{i} \cdot v_{i} \cdot e^{-\left|v_{i}-v_{i}^{\prime}\right|},
$$

where $N_{s}$ is the total number of road segments excluding the wireless chargers in road segment set and $N_{e}$ is the total number of wireless chargers. Recall that in Section 3.2, we have explained that an EV will be fully charged only when it drives through the wireless charger lane with a velocity equal to or lower than the charger lane's specified EV passing velocity $v_{i}^{\prime}$. Considering that we also expect EVs to pass through a wireless charger lane as fast as possible, we use the $e^{-\left|v_{i}-v_{i}^{\prime}\right|}$ on the utility part of wireless chargers to specify that the central controller expects the EVs to pass through each wireless charger lane at their specified EV passing velocity $v_{i}^{\prime}$. A passing velocity other than this value will result in a utility loss for the central controller.

5.3.4 Solution to the Formulated Stackelberg Game. As discussed above, we model the problem of selecting the optimal driving velocity for EVs as a Stackelberg game. In this section, we explain how the Nash equilibrium to the game is reached (i.e., the game reaches a state that the service efficiency of wireless chargers is maximized while the EV drivers are satisfied with the driving status). Recall that based on Equation (11), the central controller predicts the vehicle densities of all wireless chargers and the road segments to them. It then calculates the average estimated vehicle density of the wireless chargers and the road segments connecting them during next period of gaming: $\overline{d_{c+1}}=\sum_{k=1}^{N_{s}} d_{c+1}^{s_{k}} / N_{s}+\sum_{k=1}^{N_{e}} d_{c+1}^{s_{k}} / N_{e}$. However, the determined $\overline{d_{c+1}}$ may not be feasible for every EV in the road network, and needs to be customizable for general-purpose vehicles such as private cars. Therefore, based on $\overline{d_{c+1}}$, the central controller determines a range of expected vehicle densities that are achievable by vehicle velocity adjustment, and offers these densities to each vehicle for selection, which is defined as

$$
d_{u}=\ln (u+1) \cdot \overline{d_{c+1}}, u \in[1, \ldots, n] .
$$

We use $D=\left\{d_{1}, d_{2}, \ldots, d_{n}\right\}$ to denote the $n$ levels of expected vehicle densities for $\mathcal{T}_{c+1}$. In practice, $n$ should be at least larger than the exponential constant (i.e., $n \geqslant e \approx 2.718$ ) so that the vehicle has multiple selections around $\overline{d_{c+1}}$. The central controller notifies drivers of the $D$. If $\overline{d_{c+1}}$ leads to an increased expected vehicle density $\left(d_{u}\right)$, then it means some EVs will suffer from a higher risk of traffic congestion if they all keep their current velocity. According to Equation (15), to maintain the highest utility $F\left(v_{i}, \alpha_{i}\right)$, the EV drivers will decrease driving velocity. Otherwise, the EV drivers' risk of encountering traffic congestion will be lower, which enables the drivers to increase driving velocity to maintain the highest utility $F\left(v_{i}, \alpha_{i}\right)$. Note that the increment rate of $U_{s}(\cdot)$ (Natural Logarithmic Function) is slower than $U_{r}(\cdot)$ (product of Sigmoid Function and Linear Function) when velocity $v_{i}$ increases. Therefore, according to Equation (12), increasing driving velocity on current road segment $\left(v_{i}\right)$ will reduce a driver's utility, because $U_{r}(\cdot)$ will increase faster than $U_{s}(\cdot)$. 


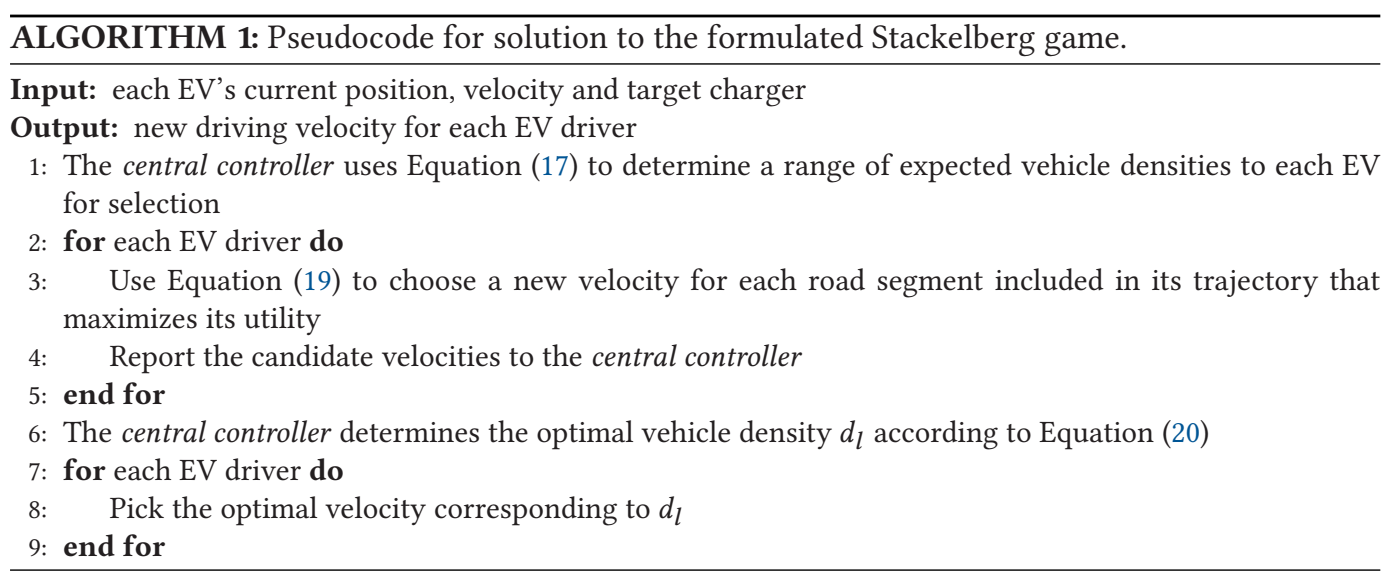

Thus, driving at a slower velocity can prevent the vehicle density of the wireless chargers and the road segments connecting them from further increasing.

For each $d_{u} \in D$, if a driver will drive in its current road segment $s_{i}$ during the next time slot, then it chooses a new velocity that maximizes its utility $F(\cdot)$, denoted by $v_{i u}$ :

$$
v_{i u}=\underset{v_{i} \leqslant v_{i}^{\max }}{\arg \max } F\left(v_{i}, \alpha_{i}\right)
$$

If a driver will drive through more than one road segment $s_{i}, s_{j}, \ldots$, then it chooses a set of velocities in each of the segments to maximize its utility $F(\cdot)$, denoted by $\left\{v_{i u}, v_{j u}, \ldots\right\}$, as shown in Equation (19),

$$
\left\{v_{i u}, v_{j u}, \ldots\right\}=\underset{v_{k} \leqslant v_{k}^{\max }}{\arg \max } \sum_{k} F\left(v_{k}, \alpha_{k}\right) .
$$

Finally, the driver reports the $n$ candidate velocities to the central controller. To maximize its utility $L(\cdot)$ based on the candidate velocities from all drivers, the central controller determines the expected vehicle density $\left(d_{l}\right)$ :

$$
d_{l}=\underset{d_{u} \in D}{\arg \max } L\left(d_{u}\right)=\underset{d_{u} \in D}{\arg \max } d_{u} \sum_{N_{s}} v_{i u}
$$

The central controller then uses the $d_{l}$ as the new expected vehicle density and notifies it to all the drivers. Then, each driver picks the optimal velocity corresponding to $d_{l}$ from the $n$ candidate velocities. The process of generating the solution to the formulated Stackelberg game is summarized in Algorithm 1.

In practice, the EV drivers may be unaware of possible inferior performance (e.g., more frequent congestion) that will be caused by driving at a sub-optimal velocity, and choose not to follow the recommended velocity. To better implement the game-theoretic framework in reality, we can refer to several approaches to motivate the EV drivers. For example, we can refer to a more interactive navigation interface, like Waze [49], Google Maps [50], or velocity planning algorithms [51, 52], to provide road alerts or energy efficiency hints that affect EV drivers' behavior. Another way is to directly adjust velocity limit of the road segments to force the EV drivers to follow the optimal velocity [53]. We leave the detailed design and implementation of such an auxiliary guidance system to our future work plans. 


\section{PERFORMANCE EVALUATION}

\subsection{Comparison Methods}

To evaluate WPT-Opt's performance, we compare it with a representative charging station recommendation system [5] (Recommend in short) and a baseline method, in which each EV selects the nearest charging station for recharge (Baseline in short). To make the methods comparable, they all use the same deployment of wireless chargers based on the existing positions of charging stations. In Recommend, when an EV sends out a request for recharging, the central controller calculates the non-charging time of each existing charging station based on current occupancy of the charging station, and the charger seeking time based on current traffic. Finally, the central controller outputs the charging station with the minimal charging time cost. While in Baseline, whenever an EV requests a recharge, the central controller recommends it the charger lane with the shortest driving time. Note that the calculation of the driving time does not consider current traffic state on road network. In addition, we also evaluate the performance of the extension of WPT-Opt (denoted as $W P T-O p t+$ ), which considers the friendship of vehicles and the drivers' routing choice based on the presence of chargers in road vehicle density calculation. In WPT-Opt and WPT-Opt+, we assume that the target charger lane of the EVs are determined by the central controller with the same method of Recommend based on current traffic status and charger availability.

\subsection{Experiment Settings}

We set the charger lane length at each charging position to be $1 \mathrm{~km}$ [2]. The battery capacities of the EVs follow a uniform distribution between $32 \mathrm{kWh}$ and $37 \mathrm{kWh}$, which is the common battery capacity of public service EVs in Shenzhen [1]. The charging rate of a charger lane is $150 \mathrm{~kW}$ [2]. This means that each EV needs around $900 \mathrm{~s}$ ( 0.25 hours) to get a full recharge. That is, the specified EV passing velocity of a wireless charger lane $\left(v_{i}^{\prime}\right.$ in Equation (16)) is $1 \mathrm{~km} / 0.25$ hours = $4 \mathrm{~km} / \mathrm{h}$. We use SUMO [54] to simulate 10,000 EVs on Shenzhen's road network for 24 hours. We set the SoC threshold to be $20 \%$. It is determined so that an EV is able to use its residual SoC to reach its nearest charger lane [1,4]. When the SoC of an EV is lower than the threshold, it will send a charging request to the central controller. We suppose that every EV starts driving with a random $\mathrm{SoC}$ value higher than the threshold at the beginning of a day. To find the best values for the parameters related to the determination of vehicle friendship (i.e., routine observation window size $N_{d}$, routine ratio threshold $\kappa$, start time deviation threshold $\sigma_{s}$, and similarity threshold $\theta$ ), we vary each parameter within a certain range and test different combinations of the parameters (e.g., [7 days, 60 days] for $N_{d}$, [0.1, 0.5] for $\kappa$, [10 minutes, 60 minutes] for $\sigma_{s}$, and $[0.2,0.6]$ for $\theta$ ). Specifically, we use each combination to determine vehicle friendship and estimate vehicle density as in Equation (11), and run our experiment for 24 hours. Then, we choose the combination of parameters that results in the minimum charging time cost. Finally, we found $N_{d}=30$ days, $\kappa=$ $0.2, \sigma_{s}=30$ minutes and $\theta=0.4$ are the best parameters for Shenzhen. The metrics we measured are as follows:

- Average non-charging time of EVs. For each EV, we measure its non-charging time at the chargers from 00:00 to 23:00. Then, we take the average non-charging time over all the EVs. We also measure the CDF of the non-charging time of each recharge. We measure this to compare the methods' performance in reducing the EVs' non-charging time.

- Average charger seeking time of EVs. For each EV, we measure its charger seeking time to its target charger from 00:00 to 23:00. Then, we take the average charger seeking time over all the EVs. We also measure the CDF of the charger seeking time of each recharge of all the EVs. We measure this metric to compare the methods' performance in reducing the EVs' charger seeking time. 


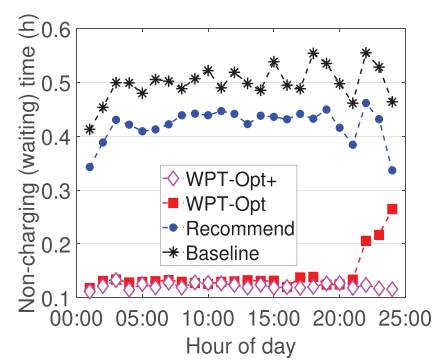

Fig. 14. Average non-charging time of all EVs per hour.

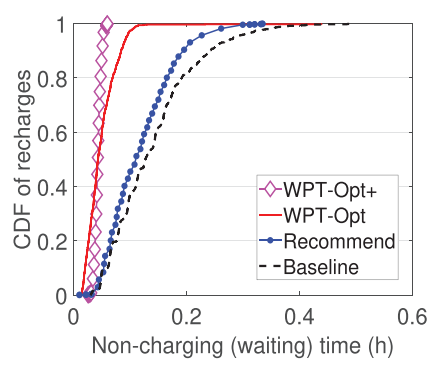

Fig. 15. Distribution of noncharging time of all EVs' recharges.

- Average number of charged EVs. We measure the total number of charged EVs per hour during a day. We also measure the CDF of the charge counts of all the EVs. We measure this metric to compare the methods' performance in maximizing the service efficiency of all wireless chargers.

- Average vehicle flow rate of all road segments. We measure the average vehicle flow rate of all road segments per hour during a day. We measure this metric to compare the methods' performance in avoiding traffic congestion.

\subsection{Experimental Results}

6.3.1 Average Non-charging Time of EVs. Figure 14 shows the average non-charging time of all the EVs per hour under different methods. Figure 15 shows the CDF of the non-charging time of each recharge of all the EVs. We can see that during most hours in Figure 14 and for most recharges in Figure 15, the results follow: WPT-Opt $+<W P T$-Opt $<$ Recommend $<$ Baseline.

Baseline always has the highest result during all times. This is because it does not consider the possible generation of congestion at the chargers after determining the target charger. When an EV arrives at a charger lane, there will usually be several other EVs that have arrived at the charger lane prior to its arrival and generate congestion. Therefore, the EV has to wait until the other congested EVs finish their recharging, which greatly increases the EV's non-charging time before recharge. This is also verified in Figure 15. We can see that the non-charging time of most recharges in Baseline (>80\%) is longer than 0.1 hours. Considering that an EV will look for a recharge whenever its SoC is below $50 \%$, and it only takes $450 \mathrm{~s}$ to recharge $50 \%$ of the EV battery, we conclude that most EVs in Baseline are influenced by congestion.

In Recommend, the average non-charging time of the EVs per hour of day and the CDF of the non-charging time are quite approximate to those in Baseline. These results demonstrate that Recommend is ineffective in preventing congestion at the chargers. This is because it makes the recommendation without considering the future change of charger availability and the traffic change on the road segments to the charger lane. Thus, its estimated future EV arrivals at the charger lane is not accurate, which may cause traffic congestion at the charger lane or on the way to the charger lane.

The EVs' non-charging time in WPT-Opt is much shorter than that in the other methods. This is because WPT-Opt can utilize the EVs' trajectories to estimate the future vehicle density at the chargers, and has a game-theoretic approach to avoid the generation of traffic congestion at the chargers and meanwhile enable the EVs to drive by their expected velocity.

In WPT-Opt+, the EVs' average non-charging time is approximate to that in WPT-Opt during most hours. In Figure 15, we can see that for WPT-Opt+, the non-charging time of more than $60 \%$ 


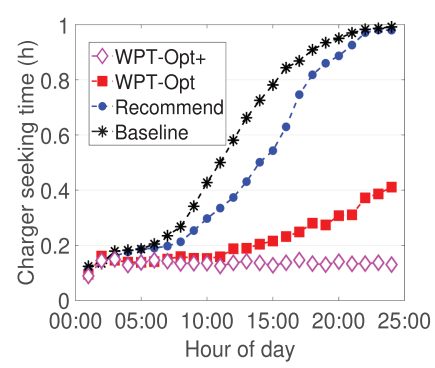

Fig. 16. Average charger seeking time of all EVs per hour.

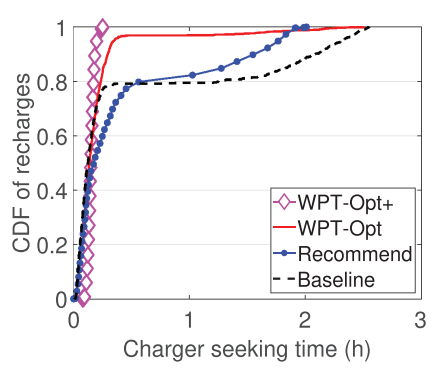

Fig. 17. Distribution of charger seeking time of all EVs' recharges.

of the recharges is shorter than that in WPT-Opt. This is because the consideration of the vehicle friendship benefits the estimation of the presence of certain vehicles that share similar driving routes during similar time periods. Therefore, the estimation of vehicle density on certain road segments is more accurate, which also makes the calculation of charging time cost and prediction of possible traffic congestion in the near future more accurate. Moreover, by considering the vehicles' routing choice and the effect of vehicles' queuing delay on charger lanes in estimating the EVs' presence probability on the road segments, the accuracy of vehicle density estimation is further increased. Thus, the EVs in WPT-Opt+ can drive to their target charger lanes with shorter non-charging time than the other methods and without suffering from traffic congestion.

6.3.2 Average Charger Seeking Time of EVs. Figure 16 shows the average charger seeking time of all the EVs per hour under different methods. We can see that during most time intervals, the results follow: WPT-Opt $+<W P T-O p t<$ Recommend $<$ Baseline. Figure 17 shows the CDF of the charger seeking time of each recharge of all the EVs. We can see that around $80 \%$ of the recharges have similar charger seeking time among different methods, but the other $20 \%$ of the recharges follow: $W P T-O p t+<W P T-O p t<$ Recommend $<$ Baseline.

$W P T-O p t$ and WPT-Opt+ always have the shortest charger seeking time. Before optimization, the future vehicle density on the predetermined driving route has been deduced by the central controller from the EVs' trajectories. Thus, WPT-Opt and WPT-Opt+ enable the central controller to maximally avoid road congestion caused by competition on certain road segments. Meanwhile, each EV can drive by a velocity as fast as possible. As a result, these two methods generate the shortest charger seeking time. Moreover, since WPT-Opt+ further considers the routing choice of the vehicles in calculating their presence probability, the vehicle density on the way from the EVs' current positions to their target charger lanes can be estimated with a higher accuracy. By driving the velocity determined based on the more accurate vehicle density, the EVs' seeking time for their target charger lanes is significantly reduced.

Recommend has the second shortest charger seeking time to target chargers during most time intervals. However, during the time intervals between 04:00 and 09:00, the EVs' charger seeking time in Recommend is even longer than that in Baseline. This is because the controller selects the route with the minimum vehicle density and the charging station with available charging point based on current vehicle density on the road network and current availability of the charging stations. Since the selected driving route and charging station are not guaranteed to be free from traffic congestion, especially during rush hours, the EVs are sometimes delayed by traffic congestion generated in the near future. 


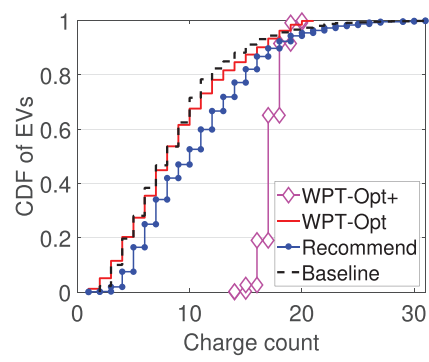

Fig. 18. Distribution of charge counts of all EVs.

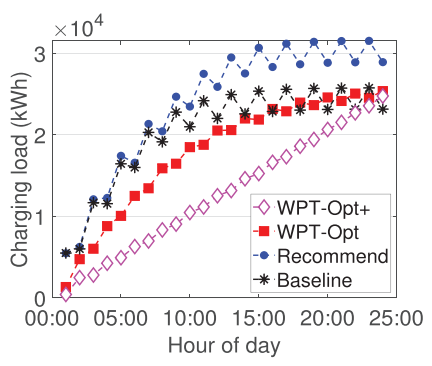

Fig. 19. Total charging load of all chargers per hour.

Baseline usually has the longest charger seeking time. This is because it does not have any approach to avoid the road segments and chargers that may suffer from traffic congestion. We can see that its charger seeking time increases enormously after 09:00, and can be as long as 2.5 hours. These results demonstrate how bad the traffic congestion can degrade the fulfillment of EVs' charging requests.

6.3.3 Average Number of Charged EVs. Figure 18 shows the CDF of the charge counts of all the EVs under different methods. We can see that for more than $90 \%$ of the EVs, the results follow: $W P T-O p t+>W P T-O p t \approx R e c o m m e n d \approx B a s e l i n e$. Figure 19 shows the total charging load created by the recharges of all the EVs per hour under different methods. We can see that by the end of the day, the total charging loads of the chargers are similar in different methods.

In WPT-Opt+, the charge counts of the EVs are quite similar (between 14 and 20), while in the other three methods, the charge counts of the EVs have much variance (between 3 and 30). This is because in the other three methods, some EVs suffered from congestion at or on the way to their target chargers, they drove much shorter distances than the other EVs and only received a few total charge counts. From Figure 19, we can see that the charging loads of WPT-Opt, Recommend and Baseline increase rapidly in the first 12 hours but more slowly in the rest 12 hours. This is because before around 12:00, there are not many EVs requesting recharge, so the EVs can be timely charged at the chargers. After 12:00, some EVs began to suffer from congestion and cannot be timely charged, so the increase of total charging load slows down during these hours. In contrast, the charging load of WPT-Opt+ increases almost uniformly by the hour. This is because the congestion has been maximally avoided, most of the EVs' recharge is timely completed. These results also show that all the methods have served the charging demands of EVs, but with different time costs. Considering that WPT-Opt+ and WPT-Opt have much shorter charging time costs, these results demonstrate that WPT-Opt+ and WPT-Opt can achieve a much higher wireless charger service efficiency.

6.3.4 Average Vehicle Flow Rate of All Road Segments. Figure 20 shows the average vehicle flow rate of all the road segments per hour under different methods. We can see the results follow: WPT$O p t+\approx W P T-O p t>$ Recommend $>$ Baseline. These results are generally consistent with those demonstrated in Figure 14 and Figure 16 due to the same reasons. It shows that WPT-Opt+ and WPT-Opt can effectively avoid the generation of traffic congestion at wireless chargers and the road segments to them.

6.3.5 Effectiveness of Considering Friendship among Vehicles and Vehicle Routing Choice Behavior. As discussed in Section 5.2.2 and Section 5.2.3, the additional consideration of the vehicles' friendship and the vehicles' routing choice behavior in Equation (11) can improve the calculation 


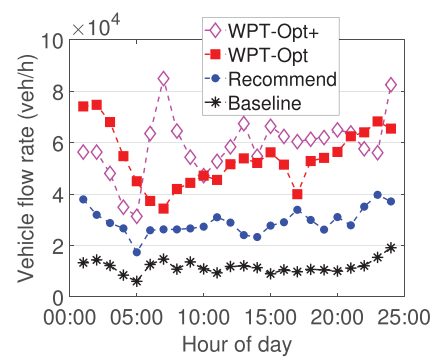

Fig. 20. Average vehicle flow rate of all road segments per hour.

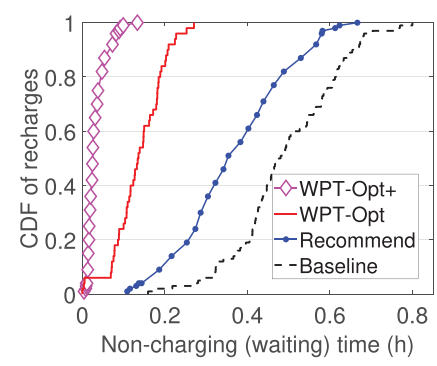

Fig. 22. Distribution of noncharging time of all EVs' recharges in Rome.

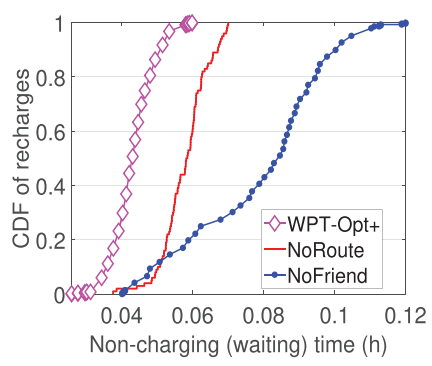

Fig. 21. Impact of components on the non-charging time of all the recharges.

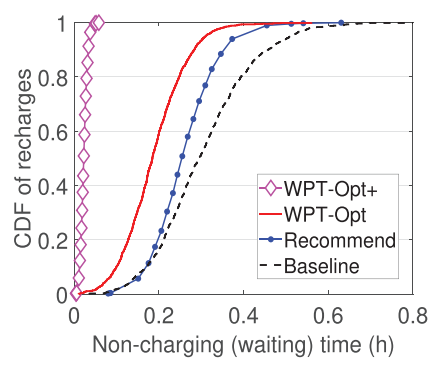

Fig. 23. Distribution of noncharging time of all EVs' recharges in San Francisco.

accuracy of the vehicle density of the road segments. To demonstrate the effectiveness of these two components in improving the service efficiency of wireless chargers and avoiding traffic congestion, we measured the CDF of the non-charging time of each recharge of all the EVs without utilizing the vehicles' friendship (denoted as NoFriend) and the vehicles' routing choice behavior (denoted as NoRoute) in gaming, respectively. The measurement results are shown in Figure 21.

We can see that the results generally follow: WPT-Opt $+<$ NoRoute $<$ NoFriend. The non-charging time of more than $80 \%$ of the recharges in NoFriend is much longer than that in the other two methods. This is because, compared with the drivers' routing choice behavior, the EVs' friendship reveals when and which road segment certain vehicles will appear and the corresponding appearance probabilities. Thus, the EVs' friendship is more reliable in estimating the vehicle density of the road segments. The results confirm the effectiveness of the two components in reducing the EVs' non-charging time.

6.3.6 Generality of WPT-Opt Approach For Different City Traffic Scenarios. To verify the generality of the proposed approach in different city traffic scenarios, we also implemented WPT-Opt using two taxicab traces from Rome and San Francisco. Specifically, the Rome Taxicab Trace is a 30-day Global Positioning System (GPS) coordinate record of 320 taxis driving in the center of Rome from February 1, 2014, to March 2, 2014. The San Francisco Taxicab Trace is a 30-day GPS coordinate record of 536 taxi traces driving in the San Francisco Bay Area from May 17, 2008, to June 15, 2008. We simulated the experiment for 30 days and measured the non-charging time of each recharge of all the EVs. Figure 22 shows the CDF of the measured results in Rome, and Figure 23 shows the measured results in San Francisco. 
We can see that similarly to Figure 15, the results follow WPT-Opt + WPT-Opt $<$ Recommend $<$ Baseline in both traces. The performance of Recommend is only slightly better than that of Baseline in both traces, which is due to the same reasons as explained in Section 6.3.1. However, compared with WPT-Opt+, which results in the shortest non-charging time in both traces, the performance of WPT-Opt in the San Francisco trace is significantly worse than that in the Rome trace. This is because Rome's road segments are quite crowded at popular sites and have short distance [55], which makes the travel time estimation of the road segments based on the vehicles' current trajectories sufficiently accurate for avoiding traffic congestion on the wireless charger lanes. While for San Francisco, the road segment distribution of San Francisco is more uniform than that in Rome [56], where the friendship information and routing choice behavior model can be effectively combined to help estimate the possible positions of the EVs, especially when the current trajectory does not cover the whole gaming period. The results confirm that WPT-Opt+ can adapt to different city traffic scenarios.

\section{CONCLUSION}

To maximize the service efficiency of wireless chargers, we must properly coordinate EVs' traffic and their arrival at the chargers to avoid traffic congestion at the chargers and on the road segments to them. Our proposed approach, WPT-Opt, is the first work that maximizes the service efficiency of wireless chargers without generating congestion, and meanwhile minimizes the EVs' charging time cost before charging. Our data analysis results confirm that EVs have spatial and temporal preference on selecting chargers, and such preferences can lead to competition for chargers. We also analyzed the relation between vehicle density and vehicles' actual driving velocity on a road segment. Moreover, we analyzed the normal distribution of travel time of road segments, and the fact that vehicles have similar frequently driven trajectories, which can be helpful in further improving the estimation accuracy of road vehicle density. Supported by these results, we formulate a non-cooperative Stackelberg game between all the EVs and a central controller, in which each EV aims at minimizing its charging time cost to its target charger lane, while the central controller tries to maximally avoid the generation of congestion on wireless chargers and the road segments to them. Our trace-driven experiments on SUMO demonstrate that WPT-Opt can maximally reduce the average charging time cost of the EVs by approximately $200 \%$ over comparison methods. In the future, we plan to consider more EV charging behavior factors (e.g., different charging time and target charger lane in weekday and weekend).

\section{REFERENCES}

[1] Li Yan, Haiying Shen, Zhuozhao Li, Ankur Sarker, John A. Stankovic, Chenxi Qiu, Juanjuan Zhao, and Chengzhong Xu. 2018. Employing opportunistic charging for electric taxicabs to reduce idle time. ACM Interact, Mobile Wear. Ubiq. Technol. 2, 1 (2018), 1-25.

[2] Y. J. Jang, E. S. Suh, and J. W. Kim. 2016. System architecture and mathematical models of electric transit bus system utilizing wireless power transfer technology. Syst. f. 10, 2 (2016), 495-506.

[3] Aristeidis Karalis, John D. Joannopoulos, and Marin Soljačić. 2008. Efficient wireless non-radiative mid-range energy transfer. Ann. Phys. 323, 1 (2008), 34-48.

[4] Li Yan, Haiying Shen, Juanjuan Zhao, Chengzhong Xu, Feng Luo, and Chenxi Qiu. 2017. CatCharger: Deploying wireless charging lanes in a metropolitan road network through categorization and clustering of vehicle traffic. In Proc. of INFOCOM.

[5] Zhiyong Tian, Taeho Jung, Yi Wang, Fan Zhang, Lai Tu, Chengzhong Xu, Chen Tian, and Xiang-Yang Li. 2016. Realtime charging station recommendation system for electric-vehicle taxis. IEEE Trans. Intell. Transport. Syst. 17, 11 (2016), 3098-3109.

[6] Zhongjing Ma, Duncan Callaway, and Ian Hiskens. 2010. Decentralized charging control for large populations of plug-in electric vehicles. In Proc. of CDC.

[7] Lingwen Gan, Ufuk Topcu, and Steven H. Low. 2013. Optimal decentralized protocol for electric vehicle charging. IEEE Trans. Power Syst. 28, 2 (2013), 940-951. 
[8] Francesco Malandrino, Claudio Casetti, and Carla-Fabiana Chiasserini. 2015. A holistic view of its-enhanced charging markets. IEEE Trans. Intell. Transport. Syst. 16, 4 (2015).

[9] Jun-Li Lu, Mi-Yen Yeh, Yu-Ching Hsu, Shun-Neng Yang, Chai-Hien Gan, and Ming-Syan Chen. 2012. Operating electric taxi fleets: A new dispatching strategy with charging plans. In Proc. of IEVC.

[10] Hua Qin and Wensheng Zhang. 2011. Charging scheduling with minimal waiting in a network of electric vehicles and charging stations. In Proc. of VIN.

[11] Hye-Jin Kim, Junghoon Lee, Gyung-Leen Park, Min-Jae Kang, and Mikyung Kang. 2010. An efficient scheduling scheme on charging stations for smart transportation. In Proc. of SEUCSG.

[12] Olle Sundström and Carl Binding. 2010. Planning electric-drive vehicle charging under constrained grid conditions. In Proc. of POWERCON.

[13] Sungwoo Bae and Alexis Kwasinski. 2012. Spatial and temporal model of electric vehicle charging demand. IEEE Trans. Smart Grid 3, 1 (2012), 394-403.

[14] Yu Zheng, Zhao Yang Dong, Yan Xu, Ke Meng, Jun Hua Zhao, and Jing Qiu. 2014. Electric vehicle battery charging/swap stations in distribution systems: Comparison study and optimal planning. IEEE Trans. Power Syst. 29, 1 (2014), 221-229.

[15] Weifeng Yao, Junhua Zhao, Fushuan Wen, Zhaoyang Dong, Yusheng Xue, Yan Xu, and Ke Meng. 2014. A multiobjective collaborative planning strategy for integrated power distribution and electric vehicle charging systems. IEEE Trans. Power Syst. 29, 4 (2014), 1811-1821.

[16] Yuchen $\mathrm{Wu}$, Yanmin Zhu, and Bo Li. 2011. Trajectory improves data delivery in vehicular networks. In Proc. of INFOCOM.

[17] Jaehoon Jeong, Shuo Guo, Yu Gu, Tian He, and David H. C. Du. 2011. Trajectory-based data forwarding for light-traffic vehicular ad hoc networks. IEEE Trans. Parallel Distrib. Syst. 22, 5 (2011), 743-757.

[18] Jaehoon Jeong, Shuo Guo, Yu Gu, Tian He, and David H. C. Du. 2012. Trajectory-based statistical forwarding for multihop infrastructure-to-vehicle data delivery. IEEE Trans. Mobile Comput. 11, 10 (2012), 1523-1537.

[19] Fulong Xu, Shuo Guo, Jaehoon Jeong, Yu Gu, Qing Cao, Ming Liu, and Tian He. 2011. Utilizing shared vehicle trajectories for data forwarding in vehicular networks. In Proc. of INFOCOM.

[20] Yu Zheng. 2015. Trajectory data mining: An overview. IEEE Trans. Intell. Syst. Technol. 6, 3 (2015), 1-41.

[21] Noortje Groot, Bart De Schutter, and Hans Hellendoorn. 2015. Toward system-optimal routing in traffic networks: A reverse stackelberg game approach. IEEE Trans. Intell. Transport. Syst. 16, 1 (2015), 29-40.

[22] OpenStreetMap. Retrieved March 2020 from http://www.openstreetmap.org/.

[23] Desheng Zhang, Jun Huang, Ye Li, Fan Zhang, Chengzhong Xu, and Tian He. 2014. Exploring human mobility with multi-source data at extremely large metropolitan scales. In Proc. of MobiCom.

[24] Yanhua Li, Jun Luo, Chi-Yin Chow, Kam-Lam Chan, Ye Ding, and Fan Zhang. 2015. Growing the charging station network for electric vehicles with trajectory data analytics. In Proc. of ICDE.

[25] Yanyan Xu, Qing-Jie Kong, Shu Lin, and Yuncai Liu. 2012. Urban traffic flow prediction based on road network model. In Proc. of ICNSC.

[26] Qing-Jie Kong, Qiankun Zhao, Chao Wei, and Yuncai Liu. 2013. Efficient traffic state estimation for large-scale urban road networks. IEEE Trans. Intell. Transport. Syst. 14, 1 (2013), 398-407.

[27] Harris Drucker, Christopher J. C. Burges, Linda Kaufman, Alex J. Smola, and Vladimir Vapnik. 1997. Support vector regression machines. In Proc. of NIPS.

[28] Ruimin Li, Huajun Chai, and Jin Tang. 2013. Empirical study of travel time estimation and reliability. Mathematical Problems in Engineering 2013, 504579 (2013), 1-9.

[29] Ruimin Li, Geoffrey Rose, and Majid Sarvi. 2006. Using automatic vehicle identification data to gain insight into travel time variability and its causes. FTRB 1945, 1 (2006), 24-32.

[30] Frank J. Massey Jr. 1951. The kolmogorov-smirnov test for goodness of fit. J. Am. Stat. Assoc. 46, 253 (1951).

[31] Li Yan, Haiying Shen, and Kang Chen. 2017. MobiT: A distributed and congestion-resilient trajectory based routing algorithm for vehicular delay tolerant networks. In Proc. of IoTDI.

[32] Mengwen Xu, Dong Wang, and Jian Li. 2016. DESTPRE: A data-driven approach to destination prediction for taxi rides. In Proc. of UbiComp.

[33] Dan Gusfield. 1997. Algorithms on Strings, Trees and Sequences: Computer Science and Computational Biology. Cambridge University Press.

[34] Michail Vlachos, George Kollios, and Dimitrios Gunopulos. 2002. Discovering similar multidimensional trajectories. In Proc. of ICDE.

[35] He Zhang, Zehua Wang, and Qinghe Du. 2018. Social-aware D2D relay networks for stability enhancement: An optimal stopping approach. IEEE Trans. Vehic. Technol. 67, 9 (2018), 8860-8874.

[36] Eyuphan Bulut and Boleslaw K. Szymanski. 2012. Exploiting friendship relations for efficient routing in mobile social networks. IEEE Trans. Parallel Distrib. Syst. 23, 12 (2012), 2254-2265. 
[37] L. Yan, H. Shen, and K. Chen. 2018. MobiT: Distributed and congestion-resilient trajectory-based routing for vehicular delay tolerant networks. IEEE/ACM Trans. Netw. 26, 3 (2018), 1078-1091.

[38] Li Yan, Haiying Shen, and Kang Chen. 2016. TSearch: Target-oriented low-delay node searching in DTNs with social network properties. IEEE/ACM Trans. Netw. 24, 6 (2016), 3841-3855.

[39] Hisatomo Hanabusa and Ryota Horiguchi. 2011. A study of the analytical method for the location planning of charging stations for electric vehicles. In Proc. of KES.

[40] Nan Jiang, Chi Xie, Jennifer C. Duthie, and S. Travis Waller. 2014. A network equilibrium analysis on destination, route and parking choices with mixed gasoline and electric vehicular flows. EURO f. Transport. Logist. 3, 1 (2014), 55-92.

[41] Raffaela Riemann, David Z. W. Wang, and Fritz Busch. 2015. Optimal location of wireless charging facilities for electric vehicles: Flow-capturing location model with stochastic user equilibrium. TRC: Emerg. Technol. 58 (2015), 1-12.

[42] Zhibin Chen, Fang He, and Yafeng Yin. 2016. Optimal deployment of charging lanes for electric vehicles in transportation networks. TRB: Methodol. 91 (2016), 344-365.

[43] Saeed D. Manshadi, Mohammad E. Khodayar, Khaled Abdelghany, and Halit Üster. 2017. Wireless charging of electric vehicles in electricity and transportation networks. IEEE Trans. Smart Grid 9, 5 (2017), 4503-4512.

[44] Zhibin Chen, Wei Liu, and Yafeng Yin. 2017. Deployment of stationary and dynamic charging infrastructure for electric vehicles along traffic corridors. TRC: Emerg. Technol. 77 (2017), 185-206.

[45] Illhoe Hwang, Young Jae Jang, Young Dae Ko, and Min Seok Lee. 2017. System optimization for dynamic wireless charging electric vehicles operating in a multiple-route environment. IEEE Trans. Intell. Transport. Syst. 19, 6 (2017), 1709-1726.

[46] Donald Gross. 2008. Fundamentals of Queueing Theory. John Wiley \& Sons.

[47] Li Yan and Haiying Shen. 2016. TOP: Vehicle trajectory based driving speed optimization strategy for travel time minimization and road congestion avoidance. In Proc. of MASS.

[48] Kenneth George Binmore. 1982. Mathematical Analysis: A Straightforward Approach. Cambridge University Press.

[49] Waze. Retrieved August 2020 from https://www.waze.com/.

[50] Google Maps. Retrieved August 2020 from https://www.google.com/maps.

[51] Grant Mahler and Ardalan Vahidi. 2014. An optimal velocity-planning scheme for vehicle energy efficiency through probabilistic prediction of traffic-signal timing. IEEE Trans. Intell. Transport. Syst. 15, 6 (2014), 2516-2523.

[52] Seyed Alireza Fayazi and Ardalan Vahidi. 2015. Crowdsourcing phase and timing of pre-timed traffic signals in the presence of queues: Algorithms and back-end system architecture. IEEE Trans. Intell. Transport. Syst. 17, 3 (2015), 870-881.

[53] Ye Li, Chengcheng Xu, Lu Xing, and Wei Wang. 2017. Integrated cooperative adaptive cruise and variable speed limit controls for reducing rear-end collision risks near freeway bottlenecks based on micro-simulations. IEEE Trans. Intell. Transport. Syst. 18, 11 (2017), 3157-3167.

[54] Daniel Krajzewicz, Jakob Erdmann, Michael Behrisch, and Laura Bieker. 2012. Recent development and applications of SUMO - simulation of urban mobility. Int. F. Appl. Sci. Math. 5, 3\&4 (2012).

[55] R. Amici, M. Bonola, L. Bracciale, P. Loreti, A. Rabuffi, and G. Bianchi. 2014. Performance assessment of an epidemic protocol in VANET using real traces. In Proc. of MoWNeT.

[56] Michał Piórkowski, Natasa Sarafijanovic-Djukic, and Matthias Grossglauser. 2009. A parsimonious model of mobile partitioned networks with clustering. In Proc. of COMSNETS.

Received March 2020; revised September 2020; accepted October 2020 